\title{
Study on Coal Methane Adsorption Behavior Under Variation Temperature and Pressure-Taking Xia-Yu-Kou Coal for Example
}

\author{
Hao Jingyuan, Li Dong, Zhang Xuemei, Ma Qinghua \\ Energy \& Chemical Engineering Research Center, Xi'an Siyuan University, Xi'an, China \\ Email address: \\ 923716217@qq.com (Hao Jingyuan)

\section{To cite this article:} \\ Hao Jingyuan, Li Dong, Zhang Xuemei, Ma Qinghua. Study on Coal Methane Adsorption Behavior Under Variation Temperature and \\ Pressure-Taking Xia-Yu-Kou Coal for Example. International Journal of Oil, Gas and Coal Engineering. Vol. 6, No. 4, 2018 , pp. 60-66. \\ doi: 10.11648/j.ogce.20180604.13
}

Received: June 25, 2018; Accepted: July 9, 2018; Published: August 1, 2018

\begin{abstract}
Based on the Langmuir volume and Langmuir pressure of Xiao-Yu-Kou \#3 coal's methane adsorption, Li's adsorption-flow equation's parameters have been calculated. LI adsorption-flow equation obtained can visually show and quantitatively calculate that how and how much variation temperature and pressure change the absorption capacity. Partial differential equation characterizes temperature has negative effects and pressure has positive effects. Therefore, under variation temperature and pressure conditions, coal adsorption capacity would have the maximum value.
\end{abstract}

Keywords: LI Adsorption-Flow Equations, Partial Differential for Temperature, Partial Differential for Pressure, Total Differential, The Maximum Value

\section{Introduction}

Coal is a porous medium and natural adsorbent with well-developed void system. Coal bed gas is usually defined as a hydrocarbon gas [1-2], which is adsorbed mainly on the surface of coal matrix particles, free in coal pores or dissolved in coal seam water. The adsorption amount of solid to gas is a function of temperature and gas pressure. In order to find out the rule easily, among the three variables-adsorption, temperature and pressure, one variable is usually fixed to determine the relationship between the other two variables. This relationship can be expressed by curves, such as adsorption isobaric and adsorption isometric lines. At constant temperature, the curve which describes the relationship between adsorption capacity and equilibrium pressure is called adsorption isotherm, and the most famous one of adsorption isotherm is the Langmuir isothermal adsorption equation. Zhang Qun and Zhong Ling-wen and others gave the Langmuir adsorption constant [3-4]. Zhang Tian-jun's research sample contains coal and shale [5]. There are also many other studies of isothermal adsorption [6-9]. In the actual situation, with the increase of the buried depth of coal reservoirs, the pressure increases and the temperature increases as well. A large number of experimental reports indicate that the adsorption properties of coal under the combined influence of temperature and pressure can be described qualitatively as follows:

1. The adsorption temperature has a negative effect on the absorption capacity of coal, which means the absorption capacity of coal decreases with the increase of temperature;

2. The adsorption pressure has a positive effect on the adsorption capacity of coal, which means the adsorption capacity of coal increases with the increase of pressure;

3. Under the combined effect of temperature and pressure, the negative effects of adsorption temperature from the adsorption capacity of coal and the positive impact of adsorption pressure from the adsorption capacity of coal are all involved in competition.

4. Under the combined effect of temperature and pressure, the adsorption capacity of coal has a maximum value $[3,10]$.

Therefore, it has a huge significance to establish a function equation related with both pressure and temperature on coal adsorption, which is used to qualitatively explain and quantitatively calculate the combined effect of pressure and temperature on coal adsorption. 


\section{LI Temperature Pressure Adsorption Equation}

\subsection{The Form of LI Temperature Pressure Adsorption Equation}

The LI temperature pressure adsorption equation [11] is originally used to solve the comprehensive effect of adsorption conditions (temperature, pressure, and properties of adsorbents) on the amount of gas adsorption, when gas molecules are adsorbed on porous media and flow in pores. The equation can be expressed as,

$$
\mathrm{V}=\frac{1}{\sqrt{M T}}\left[A+B P^{\beta} T^{1.5} \exp \left(\frac{\Delta}{T}\right)\right]
$$

In the form:

$\mathrm{V}$ is the adsorption rate under unit pressure and unit area $\left(\mathrm{cm}^{3} / \mathrm{g}\right)$.

$\mathrm{M}$ is a molecular weight, and the molecular weight of methane is 16 .

$\mathrm{T}$ is absolute temperature $(\mathrm{K})$;

$\mathrm{P}$ is pressure $(\mathrm{MPa})$;

$\mathrm{A}$ is a constant of microporous geometric shape for a fixed porous medium.

$\mathrm{B}$ is the adsorption flow coefficient, which is related to the adsorption area.

$\Delta$ is the energy difference $(\mathrm{K})$ between the lowest potential energy and the activation energy of an adsorbed molecule in the adsorbed mass flow, which mainly measures the relative influence of the adsorption temperature.

$\mathrm{B}$ is similar to a pressure parameter in Langmuir's adsorption isotherm equation, which mainly measures the relative influence of adsorption pressure.

\subsection{The Influence of Temperature Under Constant Pressure}

As the influence of pore geometry constant A is very small, the LI Temperature Pressure Adsorption Equation can be simplified [12]. In mathematics, under the condition of equal pressure, the temperature partial conductance is obtained:

$$
\left(\frac{\partial V}{\partial T}\right)_{P}=\frac{B}{\sqrt{M}} P^{\beta} \exp \left(\frac{\Delta}{T}\right)\left(1-\frac{\Delta}{T}\right)
$$

\subsection{The Influence of Pressure Under Constant Temperature}

In mathematics, the simplified LI Temperature Pressure Adsorption Equation is used to obtain partial derivative of pressure under isothermal conditions.

$$
\left(\frac{\partial V}{\partial P}\right)_{T}=\frac{B}{\sqrt{M}} T \exp \left(\frac{\Delta}{T}\right) \beta P^{\beta-1}
$$

\subsection{The Common Effect of Temperature and Pressure}

In mathematics, the common influence of temperature and pressure is to simplify the total differential of LI Temperature Pressure Adsorption Equation.

$$
d V=\frac{B}{\sqrt{M}} T \exp \left(\frac{\Delta}{T}\right) \beta P^{\beta-1} d P+\frac{B}{\sqrt{M}} P^{\beta} \exp \left(\frac{\Delta}{T}\right)\left(1-\frac{\Delta}{T}\right) d T
$$

\subsection{Calculation of LI Adsorption-Flow Equation}

As only two data sets (variable temperature and pressure) are needed, the four parameters A, B, beta and delta of the LI temperature pressure adsorption equation can be determined by means of nonlinear regression calculation [13]. Theoretically speaking, four parameters of the LI Temperature Pressure Adsorption Equation A, B, $\beta$ and $\Delta$ can be calculated and determined, If there is a sample with two temperatures or more than two temperatures for adsorption isotherms of Langmuir and Langmuir equation parameters.

\section{Data Source and Result Calculation}

The data from table-1 and table- 2 of the Xia-yu-kou coal No.3 are derived from the article 'The Effect of Temperature on the Adsorbing Capability of Coal' published by Zhang Tianjun in 2009 in 'Journal of China Coal Society' [5].

Table 1. Data and parameters of coal sample.

\begin{tabular}{ll}
\hline Parameters of Coal sample & $\begin{array}{l}\text { No.3 coal in Xia-yu-kou coal mine in Hancheng mining area, Shaanxi. } \\
\text { Ash content } 17.37 \% \text {; water content } 1.72 \% ; \text { R0 2.1\% }\end{array}$ \\
\hline
\end{tabular}

Table 2. The actual condition, the Langmuir volume and equation parameter of No. 3 coal sample at Xia-yu-kou coal. Mine.

\begin{tabular}{lll}
\hline Experimental Temperature $/{ }^{\circ} \mathbf{C}$ & Langmuir Volume $/ \mathbf{c m}^{\mathbf{3}} \mathbf{g}^{-1}$ & Langmuir Equation Parameters b/MPa $^{-1}$ \\
\hline 20 & 30.2 & 0.54 \\
30 & 23.5 & 0.61 \\
40 & 17.2 & 0.57 \\
50 & 14.1 & 0.58 \\
\hline
\end{tabular}

the data of table 2 is substituted into the Langmuir adsorption isotherm, the equation (5) is as followed:

$$
V=\frac{a b P}{1+b P}
$$

In the form:

$\mathrm{V}$ is the adsorption amount, $\mathrm{cm}^{3} / \mathrm{g}$; $\mathrm{A}=\mathrm{V}_{\mathrm{L}}$ is the Langmuir's volume, $\mathrm{cm}^{3} / \mathrm{g}$;

$\mathrm{P}$ is pressure, $\mathrm{MPa}$;

$\mathrm{B}=1 / \mathrm{PL}$ is the reciprocal of Langmuir's pressure, $\mathrm{MPa}^{-1}$;

As the adsorption capacity of different pressures under 4 
different temperatures conditions are obtained, and the data set under variable temperature and variable pressure conditions can be obtained.

The regression constants $\mathrm{A}, \mathrm{B}, \beta$ and $\Delta$ of the LI Adsorption-flow Equation are calculation by nonlinear regression. The results are listed in table-3.

Table 3. the parameters of the Xia-yu-kou No. 3 coal samples obtained from the LI temperature pressure adsorption equation based on the data of table. 2 .

\begin{tabular}{ll}
\hline Regression Parameter & Regression Value \\
\hline A & 0.168 \\
B & 0.0000136 \\
$\beta$ & 0.40352 \\
$\Delta$ & 2724 \\
\hline
\end{tabular}

Table 3 shows that in the papers published by Zhang Tianjun and others, the test pressure is less than $8 \mathrm{MPa}$. In order to discuss the changing theoretical calculation value of the coal's adsorption amount under variable temperature and pressure conditions, the parameter calculation of LI temperature pressure adsorption equation in table- 3 is within the range of test temperature $\left(20-50^{\circ} \mathrm{C}\right)$ and the range of test pressure (0.5-15 MPa). The maximum calculation pressure is $15 \mathrm{MPa}$.

\subsection{Temperature Pressure Adsorption Surface of No. 3 Coal Sample from Xia-yu-kou}

The temperature pressure adsorption surface is shown as figure 1 , according to equation (1) and the parameters from Table 3 .

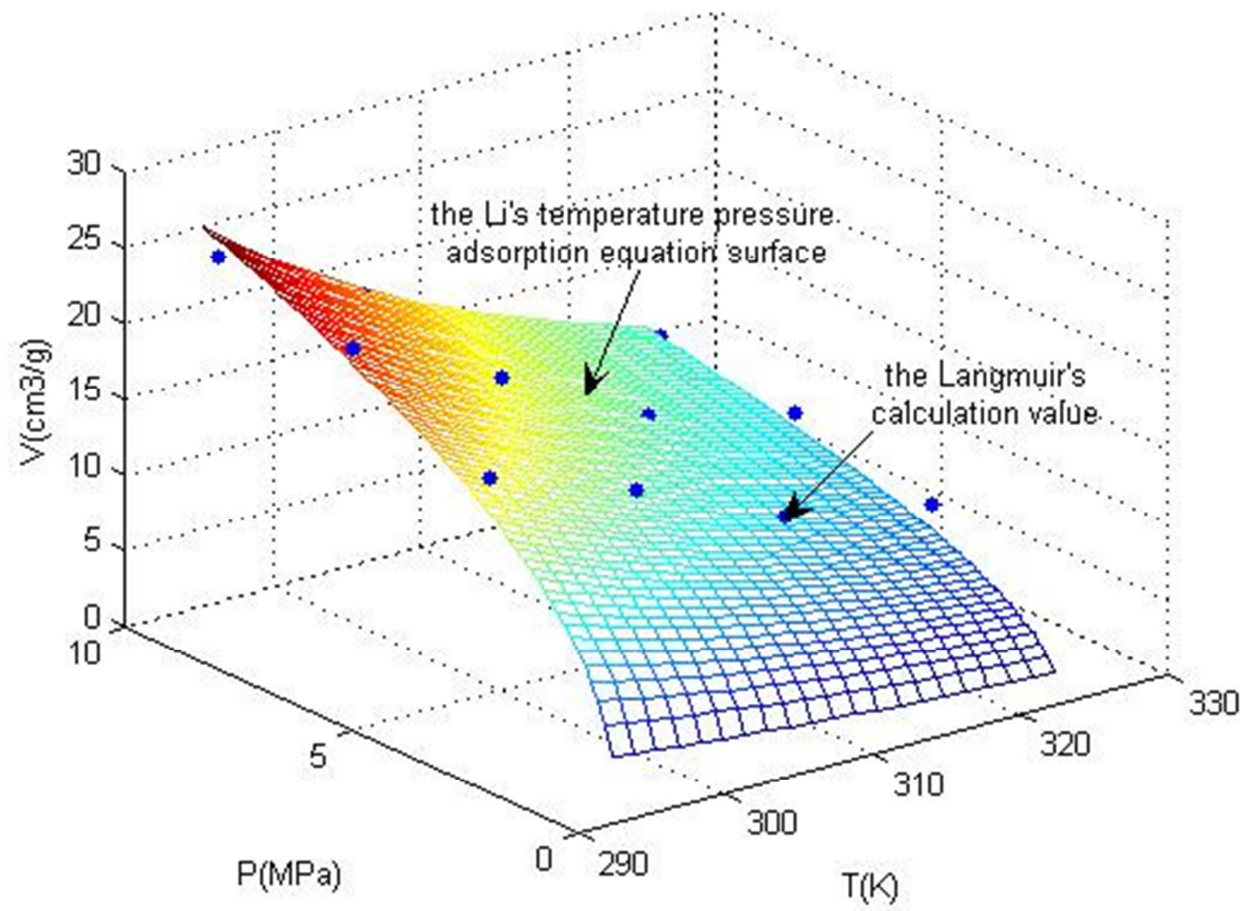

Figure 1. The Li's temperature pressure adsorption equation surface of No.3 Xia-yu-kou coal sample under the conditions of temperature 290-330 (K)and pressure 0-10 MPa. The points in figure-1 is "the Langmuir's calculation value".

Two aspects can be explained from Figure 1:

Firstly, the LI temperature-pressure-adsorption 3D view surface shows the combined effect of temperature and pressure on the adsorption capacity. Low temperature and high pressure are beneficial to the adsorption of coal bed gas (dense line area), and high temperature and low pressure are detrimental to the adsorption of coal bed gas (line evacuation area). The changes of temperature and pressure are continuous and uninterrupted; On the other hand, from figure 1, It also verifies that the relative average error between the "Langmuir's calculation value"(point) and the "LI calculation value"(surface) is very small, the reason is that the "Langmuir's calculation value" coincides with the LI temperature pressure adsorption surface.

Table 4. The comparison between Langmuir's calculation value and LI calculation value of No. 3 Xia-yu-kou coal sample.

\begin{tabular}{llll}
\hline Temperature /K & pressure/MPa & Langmuir's adsorptive capacity & Li's adsorptive capacity \\
\hline 293.15 & 6 & 23.08 & 22.13 \\
293.15 & 9 & 25.05 & 26.06 \\
303.15 & 3 & 15.20 & 12.73 \\
303.15 & 9 & 19.88 & 19.84 \\
313.15 & 3 & 10.85 & 9.87 \\
313.15 & 6 & 13.31 & 13.06 \\
323.15 & 3 & 8.95 & 7.78 \\
323.15 & 6 & 10.95 & 10.30 \\
323.15 & 9 & 11.83 & 12.13 \\
\hline
\end{tabular}


The description of table-4: the dimension of Langmuir's adsorption capacity and the amount of Li's adsorption capacity is $\mathrm{cm}^{3} / \mathrm{g}$; the Langmuir's adsorption capacity is calculated according the equation (5) based on the data from table-2; the amount of Li's adsorption capacity is calculated according to the equation (1) based on the data from table-3. Significance digit keep the two bits after the decimal point.

\subsection{The Influence of Temperature on the Adsorption \\ Capacity for Coal}

For the Xia-yu-kou No.3 coal sample, The energy difference between the lowest potential energy and the activation energy of an adsorbed molecule in the adsorbate flow is 2724 . During the test temperatures, $\left(1-\frac{\Delta}{T}\right)$ is less than zero, which is negative, shown as $\left(\frac{\partial V}{\partial T}\right)_{P}$ partial derivative of temperature in table-5. The right side of equation (2) is less than zero, and the change of adsorption capacity has a negative impact from temperature changes. Figure 2 shows that $\left(\frac{\partial V}{\partial T}\right)_{P}$, partial derivative of temperature, is negative in the range of test temperature $\left(20^{\circ} \mathrm{C}-50^{\circ} \mathrm{C}\right)$ and test pressure (0.5-15 MPa).

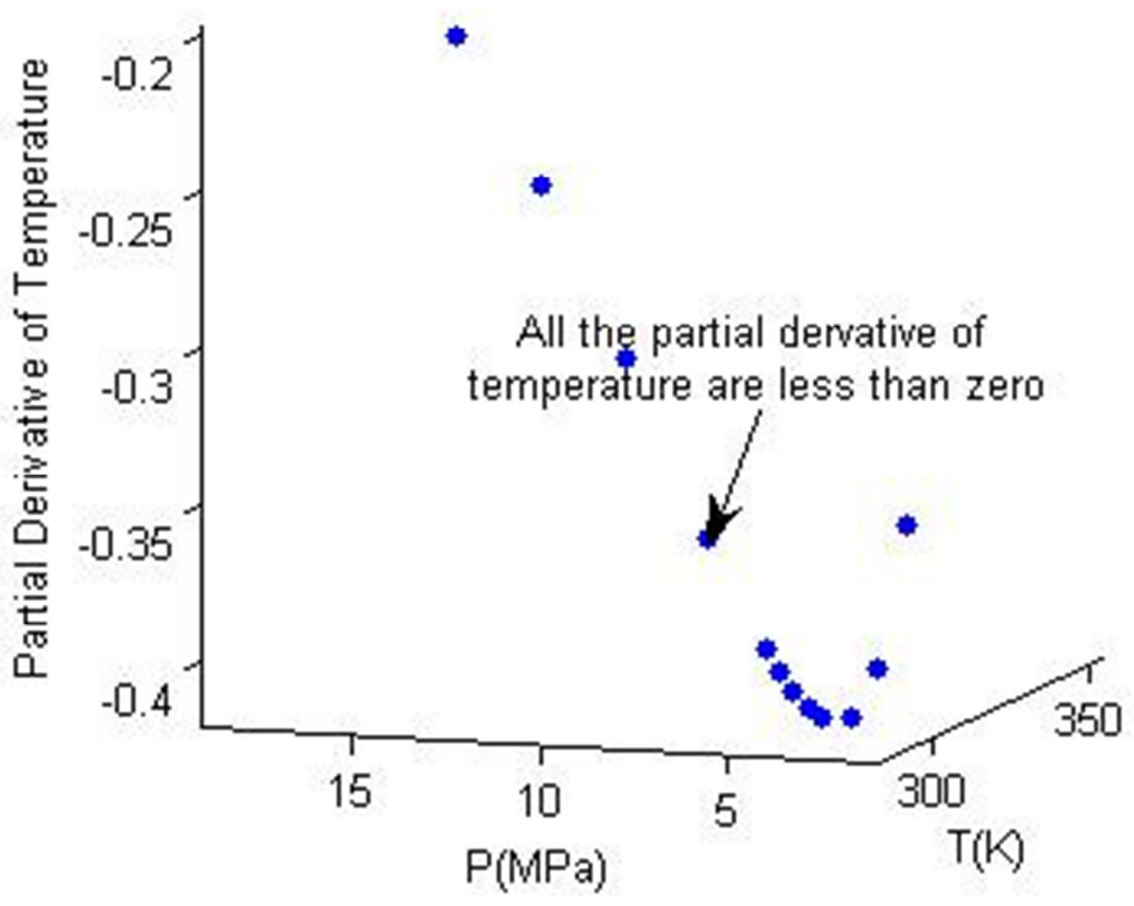

Figure 2. The calculated value of partial derivative of temperature $\left(\frac{\partial V}{\partial T}\right)_{P}$ in condition of temperature $\left(20^{\circ} \mathrm{C}-50^{\circ} \mathrm{C}\right)$ and pressure $(0.5-15 \mathrm{MPa})$ in Xiayoukou No.3 coal sample

\subsection{The Influence of Pressure on the Adsorption Capacity for Coal}

For the No. 3 coal sample of the Xia-yu-kou coal mine, the Pressure influence parameters $\beta$ of the LI temperature-pressure-adsorption equation is 0.40352 , which is greater than zero, as shown in the list of partial derivative of pressure $\left(\frac{\partial V}{\partial P}\right)_{T}$ in table-5. It is proved that the right of the equation (3) is always positive. Adsorption pressure has a positive effect on the adsorption capacity of coal under isothermal conditions. Figure 3 shows that the partial derivative of pressure is positive in the range of test temperature $\left(20^{\circ} \mathrm{C}-50^{\circ} \mathrm{C}\right)$ and test pressure $(0.5-15 \mathrm{MPa})$. 


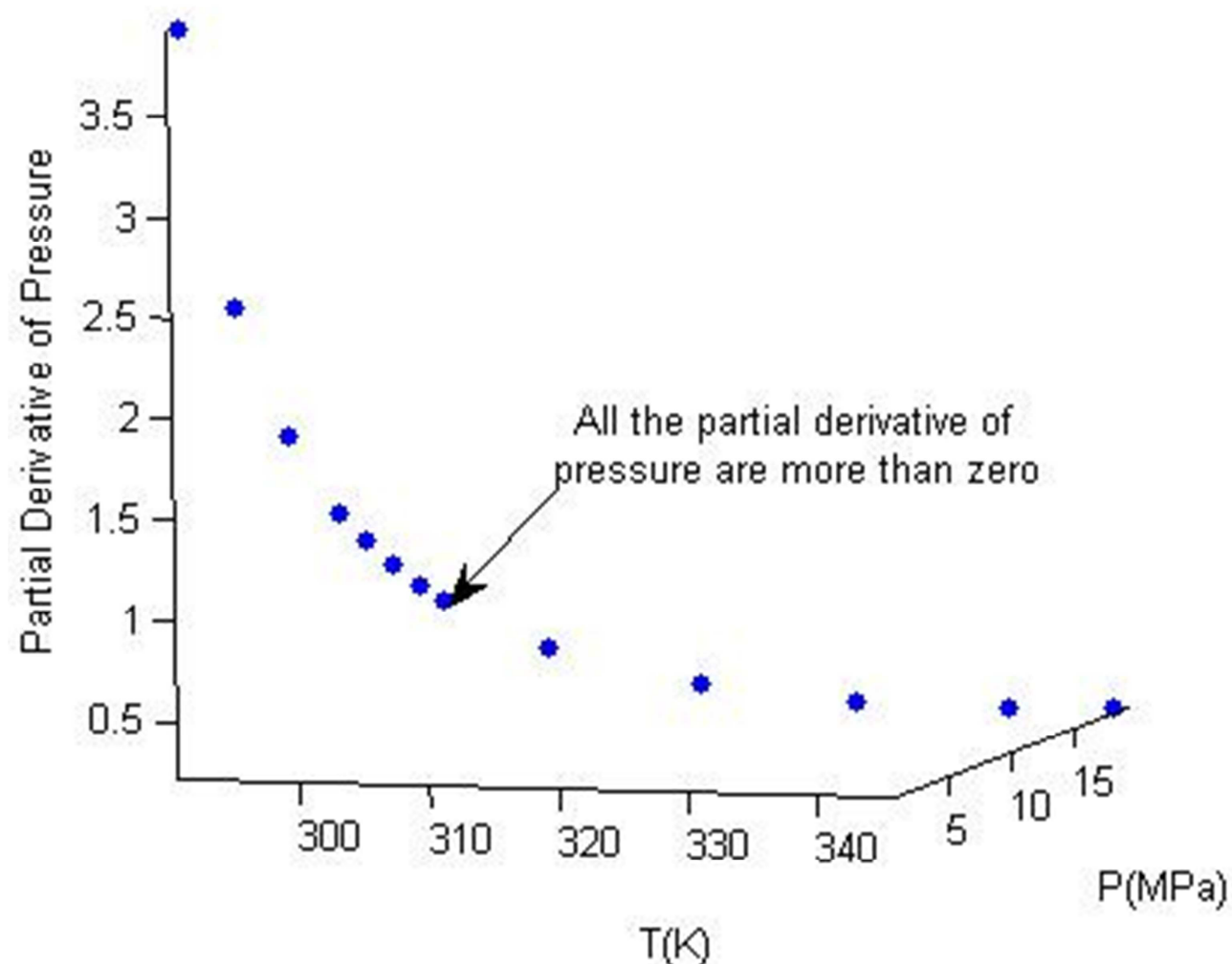

Figure 3. The calculated value of Xia-yu-kou No.3 coal sample's pressure partial derivative in the range of test temperature $\left(20^{\circ} \mathrm{C}-50^{\circ} \mathrm{C}\right)$ and test pressure (0.5-15 MPa).

Table 5. Calculated result of adsorption variation for Xia-yu-kou No.3 coal under variable temperature and variable pressure.

\begin{tabular}{|c|c|c|c|c|c|c|c|c|}
\hline T1 & $\mathbf{T} 2$ & P1 & $\mathbf{P 2}$ & $\left(\frac{\partial \boldsymbol{V}}{\partial \boldsymbol{T}}\right)_{P}$ & $\left(\frac{\partial \boldsymbol{V}}{\partial \boldsymbol{P}}\right)_{T}$ & dT & dP & $d V$ \\
\hline 293.15 & 303.15 & 3 & 3 & -0.399 & 1.947 & 10 & 0 & -3.986 \\
\hline 313.15 & 313.15 & 3 & 6 & -0.286 & 1.035 & 0 & 3 & 3.106 \\
\hline
\end{tabular}

Explanation for table-5: equation (2) is used to calculate $\left(\frac{\partial V}{\partial T}\right)_{P}$; equation (3) is used to calculate $\left(\frac{\partial V}{\partial P}\right)_{T}$; equation

(4) is used to calculate dV. T, P, dT and dP used in equations (2), (3) and (4) come from the equations as followed:

$$
\begin{gathered}
T=\frac{T_{1}+T_{2}}{2} \\
P=\frac{P_{1}+P_{2}}{2} \\
d T=T_{2}-T_{1} \\
d P=P_{2}-P_{1}
\end{gathered}
$$

\subsection{Theoretical Calculated Values of Coal's Adsorption Capacity Under Variable Temperature and Pressure}

Under the combined effect of temperature and pressure, the negative influence from adsorption temperature to adsorption capacity of coal and the positive effect from adsorption pressure to adsorption capacity of coal are all involved in the competition. The data in table- 6 shows this interaction, that is, the adsorption variation. Figure 4 shows this relationship.

Both table 6 and figure 4 show a very important information, that is, under a certain temperature and a certain pressure, the adsorption variation of coal appears a qualitative change, from the positive to negative. This is called the inflection point in mathematics. For Xia-yu-kou No.3 coal sample, the temperature of its inflection point should be between $301.65 \mathrm{~K}$ and $304.65 \mathrm{~K}$, and the pressure of that point should be between 4.5 Mpa and 5.5 MPa. 


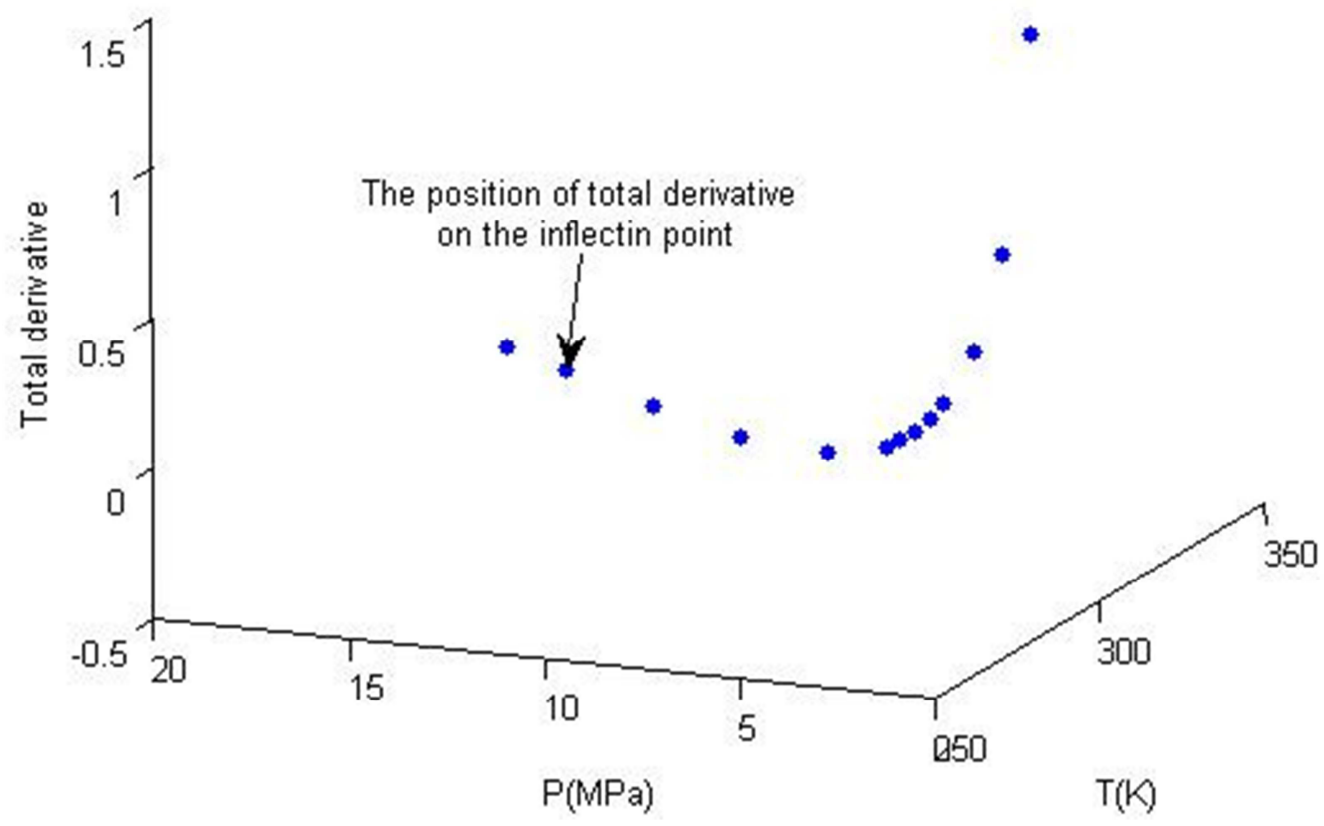

Figure 4. The total differential (adsorption variation) value of Xia-yu-kou No.3 coal sample at temperature (20 -50 C) and pressure (0.5-15 MPa).

Table 6. The calculated value of adsorption variation at inflection point for Xia-yu-kou No.3 coal under variable temperature and pressure condition.

\begin{tabular}{lllllllll}
\hline T1 & T2 & P1 & P2 & $\left(\frac{\partial \boldsymbol{V}}{\partial \boldsymbol{T}}\right)_{\boldsymbol{P}}$ & $\left(\frac{\partial \boldsymbol{V}}{\partial \boldsymbol{P}}\right)_{\boldsymbol{T}}$ & $\mathbf{d T}$ & $\mathbf{d P}$ & $\mathbf{d V}$ \\
\hline 300.15 & 301.65 & 4 & 4.5 & -0.417 & 1.467 & 1.5 & 0.5 & 0.107808 \\
301.65 & 303.15 & 4.5 & 5 & -0.415 & 1.319 & 1.5 & 0.5 & 0.037327 \\
303.15 & 304.65 & 5 & 5.5 & -0.411 & 1.194 & 1.5 & 0.5 & -0.01898 \\
304.65 & 306.15 & 5.5 & 6 & -0.405 & 1.087 & 1.5 & 0.5 & -0.0643 \\
\hline
\end{tabular}

Mathematically, it is not difficult to prove that if the adsorption variation appears an inflection point and changes from the positive to negative, it is indicated that adsorption variation must have extreme value on that inflection point, and it is a maximum. Figure 5 shows that under the dual influence of temperature and pressure, the coal adsorption capacity of the No. 3 coal sample from Xia-yu-kou coal has a maximum value.

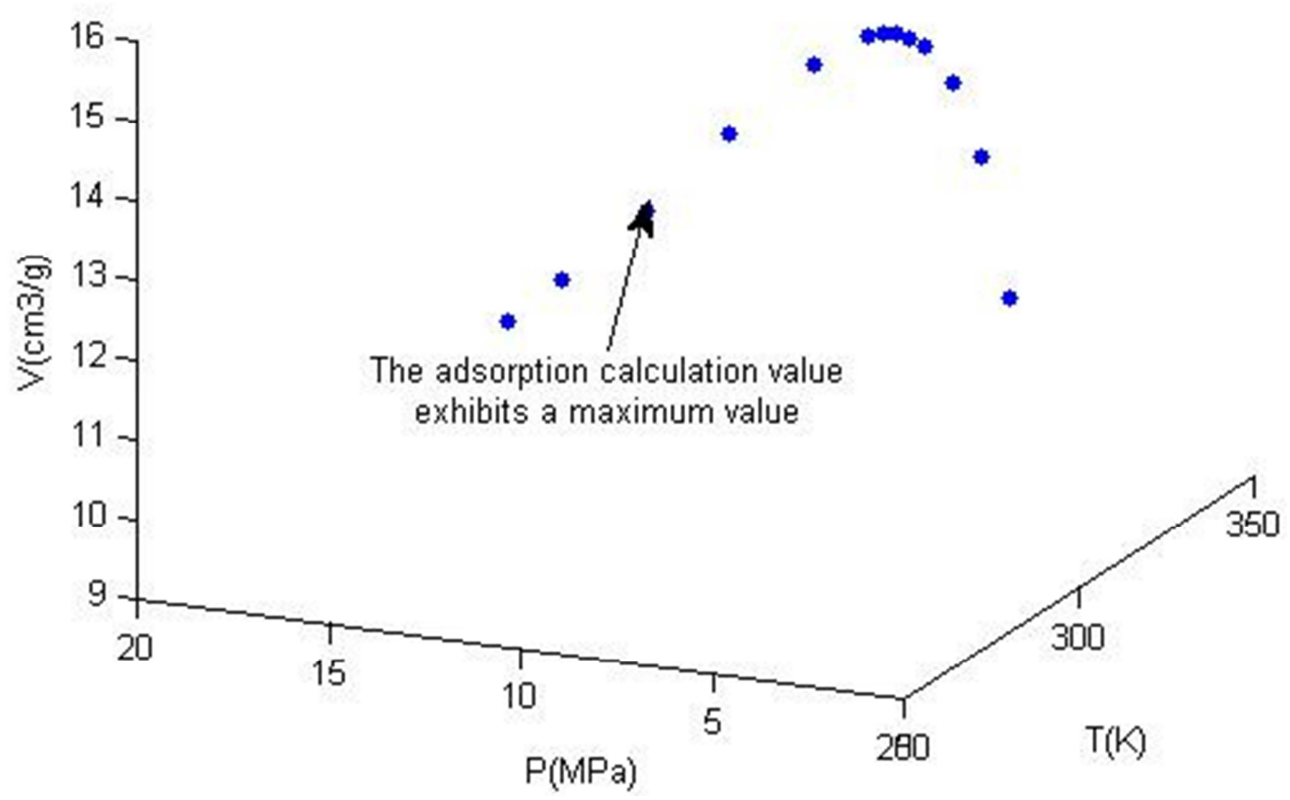

Figure 5. The adsorption calculated value of Xia-yu-kou No.3 coal sample in the range of temperature $\left.20^{\circ} \mathrm{C}-50^{\circ} \mathrm{C}\right)$ and pressure $(0.5-15 \mathrm{MPa})$. 


\section{Conclusion}

The LI temperature pressure adsorption equation determines the functional relationship between the adsorption medium, pressure and influence of temperature on adsorption. As long as there is enough adsorption data under variable temperature and pressure, the corresponding parameters of the LI temperature pressure adsorption equation can be obtained by regression calculation.

The LI temperature pressure adsorption equation can be visualized and quantitatively calculated. In the range of teat temperature and pressure, the adsorption capacity of coal decreases with the increasing temperature and increases with the increasing pressure. Under the dual influence of temperature and pressure, the adsorption capacity of coal has a maximum value.

\section{References}

[1] DAI Jin-xing, NI Yun-yan, HUANG Shi-peng, et al Significant Function of Coal-derived Gas Study for Natural Gas Industry Development in China [J], Natural Gas Geoscience, 2014, 25(01):1-22.

[2] QIN Yong, TANG Da-zhen, LIU Da-meng, et al. Geologic Evaluation Theory and Technology Progress of Coal Reservoir Dynamics During Coalbed Methane Drainage [J], Coal Science and Technology, 2014, 42(1):80-88

[3] ZHANG Qun, CUI Yong-jun, ZHONG Ling-wen, et al. Temperature pressure integrated adsorption model of methane adsorbed by coal $[\mathrm{J}]$, Journal of China Coal Society, 2008(11):1272-1278.

[4] ZHONG Lin-wen, ZHENG Yu-zhu, YUN Zheng-rong, et al. The adsorption capability of coal under integrated influence of temperature and pressure and prediction of content quantity of coalbed gas [J], Journal of China Coal Society. 2002,27(6): $581-585$

[5] ZHANG Tian-jun, XU Hong-jie, LI Shu-gang, et al. The effect of temperature on the adsorbing capacity [J], Journal of China Coal Society. 2009,34(6):802-805

[6] MA Dong-ming, ZHANG Sui-an, LIN Ya-bing, Isothermal adsorption and desorption experiment of coal and experimental results accuracy fitting $[\mathrm{J}]$, Journal of China Coal Society. 2011,36(3):477—479
[7] TANG Shu-heng, FAN Er-ping, ZHANG Song-hang, et al. Reservoir characteristics and gas-bearing capacity of the Lower Palaeozoic marine shales in Northwestern Hunan [J], Earth Science Frontiers, 2016, 23(02), 135-146

[8] ZHAO Li-juan, QIN Yong, Geoff WANG, et al. Adsorption Behavior of Deep Coalbed Methane Under High Temperature and Pressure [J], Geological Journal of China Universities, 2013,19(4):648-654

[9] XUE Jing-zhan, FU Xue-hai, FAN Chun-jie, et al. Adsorption and adsorption model of $\mathrm{H}_{2} 2 \mathrm{~S}$ in different coal ranks [J], Coal Geology \& Exploration, 2016, 44(06), 75-78

[10] ZHANG Qun, SANG Shu-xun, Adsorption characteristic and storage mechanism of coal seam, Scientific Publish House, Beijing, 2013 6-7, 110-117

[11] D. Li, Preparation and characterization of silicon base inorganic membrane for gas separation [D], University of Cincinnati, USA 1991

[12] LI Dong, CHEN Wen, FENG Xi-wen, et al. Comparative study between the measured and calculated values of coal isothermal adsorption Langmuir [J]. Shaanxi Coal, 2017, 36(02), 10-13

[13] LI Dong, ZHANG Xue-mei, CHENG Shi, et al. The influence of both temperature and pressure on the adsorption and activated flow of coalbed methane in coal seams [J], Inner Mongolia Petrochemical Industry, 2015 3: 6-8

\section{Biography}

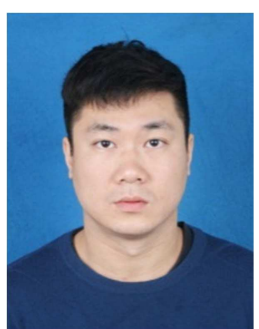

Hao Jingyuan (1989-), male, master of University of Wollongong in Australia, researcher of Energy \& Chemical Engineering Research Center in Xi'an Siyuan University, mainly engaged in the research of data processing, chemical technology and equipment, simulation. 